\title{
An Upper Bound for the Dimension of Bounded Derived Categories ${ }^{*} \dagger$
}

\author{
Junling Zheng ${ }^{a, b}$, Zhaoyong Huang ${ }^{b, \ddagger}$ \\ ${ }^{a}$ Department of Mathematics, China Jiliang University, Hangzhou 310018, Zhejiang Province, P.R. China \\ ${ }^{b}$ Department of Mathematics, Nanjing University, Nanjing 210093, Jiangsu Province, P.R. China
}

\begin{abstract}
Let $\Lambda$ be an artin algebra. We give an upper bound for the dimension of the bounded derived category of the category $\bmod \Lambda$ of finitely generated right $\Lambda$-modules in terms of the projective and injective dimensions of certain class of simple right $\Lambda$-modules as well as the radical layer length of $\Lambda$. In addition, we give an upper bound for the dimension of the singularity category of $\bmod \Lambda$ in terms of the radical layer length of $\Lambda$.
\end{abstract}

\section{Introduction}

Given a triangulated category $\mathcal{T}$, Rouquier introduced in [19] the dimension $\operatorname{dim} \mathcal{T}$ of $\mathcal{T}$ under the idea of Bondal and van den Bergh in [6]. This dimension and the infimum of the Orlov spectrum of $\mathcal{T}$ coincide, see [3, 16]. Roughly speaking, it is an invariant that measures how quickly the category can be built from one object. Many authors have studied the upper bound of $\operatorname{dim} \mathcal{T}$, see $[3,5,17,9,13,15,18,19]$ and so on. There are a lot of triangulated categories having infinite dimension, for instance, Oppermann and Št'ovíček proved in [15] that all proper thick subcategories of the bounded derived category of finitely generated modules over a Noetherian algebra containing perfect complexes have infinite dimension.

Let $\Lambda$ be an artin algebra. Let $\bmod \Lambda$ be the category of finitely generated right $\Lambda$-modules and let $D^{b}(\bmod \Lambda)$ and $D_{s g}^{b}(\bmod \Lambda)$ be the bounded derived category and singularity category of $\bmod \Lambda$ respectively. The upper bounds for the dimensions of these two categories can be given in terms of the Loewy length $\operatorname{LL}(\Lambda)$ and the global dimension gl.dim $\Lambda$ of $\Lambda$.

Theorem 1.1. Let $\Lambda$ be an artin algebra. Then we have

(1) ([19, Proposition 7.37]) $\operatorname{dim} D^{b}(\bmod \Lambda) \leqslant \operatorname{LL}(\Lambda)-1$;

(2) $\left(\left[19\right.\right.$, Proposition 7.4] and [13, Proposition 2.6]) $\operatorname{dim} D^{b}(\bmod \Lambda) \leqslant \operatorname{gl} \cdot \operatorname{dim} \Lambda$;

(3) ([5, Lemma 4.5]) $\operatorname{dim} D_{s g}^{b}(\bmod \Lambda) \leqslant \operatorname{LL}(\Lambda)-2$.

\footnotetext{
*2010 Mathematics Subject Classification: 18E30, 16E10, 16S90.

${ }^{\dagger}$ Keywords: Dimensions, Bounded derived categories, Upper bounds, Projective dimension, Injective dimension, Radical layer length.

${ }^{\ddagger}$ Email: zjlshuxue@163.com, huangzy@nju.edu.cn
} 
By Theorem 1.1(1)(3), we have that both $\operatorname{dim} D^{b}(\bmod \Lambda)$ and $\operatorname{dim} D_{s g}^{b}(\bmod \Lambda)$ are finite; however, Theorem 1.1](2) does not provide any information when gl.dim $\Lambda$ is infinite.

For a length-category $\mathcal{C}$, generalizing the Loewy length, Huard, Lanzilotta and Hernández introduced in [10, 11] the (radical) layer length associated with a torsion pair, which is a new measure for objects of $\mathcal{C}$. Let $\Lambda$ be an artin algebra and $\mathcal{V}$ a set of some simple modules in $\bmod \Lambda$. Let $t_{\mathcal{V}}$ be the torsion radical of a torsion pair associated with $\mathcal{V}$ (see Section 3 for details). We use $\ell \ell^{t_{\mathcal{V}}}(\Lambda)$ to denote the $t_{\mathcal{V}}$-radical layer length of $\Lambda$. For a module $M \operatorname{in} \bmod \Lambda$, we use $\operatorname{pd} M$ and id $M$ to denote the projective and injective dimensions of $M$ respectively; in particular, set $\operatorname{pd} M=-1=$ id $M$ if $M=0$. For a subclass $\mathcal{B}$ of $\bmod \Lambda$, the projective dimension pd $\mathcal{B}$ of $\mathcal{B}$ is defined as

$$
\operatorname{pd} \mathcal{B}= \begin{cases}\sup \{\operatorname{pd} M \mid M \in \mathcal{B}\}, & \text { if } \mathcal{B} \neq \varnothing \\ -1, & \text { if } \mathcal{B}=\varnothing\end{cases}
$$

Dually, the injective dimension id $\mathcal{B}$ of $\mathcal{B}$ is defined. Note that $\mathcal{V}$ is a finite set. So, if each simple module in $\mathcal{V}$ has finite projective (resp. injective) dimension, then $\operatorname{pd} \mathcal{V}$ (resp. id $\mathcal{V}$ ) attains its (finite) maximum.

The aim of this paper is to prove the following

Theorem 1.2. (Theorems 3.12 and 3.14) Let $\Lambda$ be an artin algebra and $\mathcal{V}$ a set of some simple modules in $\bmod \Lambda$ with $\ell^{t} \mathcal{v}(\Lambda)=n$. Then we have

(1) if $d=\min \{\operatorname{pd} \mathcal{V}$, id $\mathcal{V}\}$, then $\operatorname{dim} D^{b}(\bmod \Lambda) \leqslant(d+2)(n+1)-2$;

(2) $\operatorname{dim} D_{s g}^{b}(\bmod \Lambda) \leqslant \max \{0, n-2\}$.

In Section 3, we give the proof of Theorem 1.2. In fact, Theorem 1.1 is some special cases of Theorem 1.2 (see Remark 3.16). Moreover, by choosing some suitable $\mathcal{V}$ and applying Theorem 1.2. we may obtain more precise upper bounds for $\operatorname{dim} D^{b}(\bmod \Lambda)$ and $\operatorname{dim} D_{s g}^{b}(\bmod \Lambda)$ than that in Theorem 1.1. We give in Section 4 two examples to illustrate this and that the difference between the upper bounds obtained from Theorems 1.1 and 1.2 may be arbitrarily large.

\section{Preliminaries}

\subsection{The dimension of a triangulated category}

We recall some notions from [14, 18, 19]. Let $\mathcal{T}$ be a triangulated category and $\mathcal{I} \subseteq$ Ob $\mathcal{T}$. Let $\langle\mathcal{I}\rangle$ be the full subcategory consisting of $\mathcal{T}$ of all direct summands of finite direct sums of shifts of objects in $\mathcal{I}$. Given two subclasses $\mathcal{I}_{1}, \mathcal{I}_{2} \subseteq \mathrm{Ob} \mathcal{T}$, we denote $\mathcal{I}_{1} * \mathcal{I}_{2}$ by the full subcategory of all extensions between them, that is,

$$
\mathcal{I}_{1} * \mathcal{I}_{2}=\left\{X \mid X_{1} \longrightarrow X \longrightarrow X_{2} \longrightarrow X_{1}[1] \text { with } X_{1} \in \mathcal{I}_{1} \text { and } X_{2} \in \mathcal{I}_{2}\right\}
$$

Write $\mathcal{I}_{1} \diamond \mathcal{I}_{2}:=\left\langle\mathcal{I}_{1} * \mathcal{I}_{2}\right\rangle$. Then $\left(\mathcal{I}_{1} \diamond \mathcal{I}_{2}\right) \diamond \mathcal{I}_{3}=\mathcal{I}_{1} \diamond\left(\mathcal{I}_{2} \diamond \mathcal{I}_{3}\right)$ for any subclasses $\mathcal{I}_{1}, \mathcal{I}_{2}$ and $\mathcal{I}_{3}$ of $\mathcal{T}$ by the octahedral axiom. Write

$$
\langle\mathcal{I}\rangle_{0}:=0,\langle\mathcal{I}\rangle_{1}:=\langle\mathcal{I}\rangle \text { and }\langle\mathcal{I}\rangle_{n+1}:=\langle\mathcal{I}\rangle_{n} \diamond\langle\mathcal{I}\rangle_{1} \text { for any } n \geqslant 1
$$


Definition 2.1. ([19, Definiton 3.2]) The dimension $\operatorname{dim} \mathcal{T}$ of a triangulated category $\mathcal{T}$ is the minimal $d$ such that there exists an object $M \in \mathcal{T}$ with $\mathcal{T}=\langle M\rangle_{d+1}$. If no such $M$ exists for any $d$, then we set $\operatorname{dim} \mathcal{T}=\infty$.

Lemma 2.2. ([17, Lemma 7.3]) Let $\mathcal{T}$ be a triangulated category and let $X, Y$ be two objects of T. Then

$$
\langle X\rangle_{m} \diamond\langle Y\rangle_{n} \subseteq\langle X \oplus Y\rangle_{m+n}
$$

for any $m, n \geqslant 0$.

Lemma 2.3. ([1, Proposition 3.2]) Let $\mathcal{A}$ be an abelian category admitting enough projective objects. Let $X=\left(X^{i}, d^{i}\right)$ be a bounded complex in $\mathcal{A}$ such that the homology $H^{i}(X)$ has projective dimension at most $n$ for all $i$. Then $X \in\langle\mathcal{P}\rangle_{n+1} \subseteq D^{b}(\mathcal{A})$ for the subcategory $\mathcal{P} \subseteq \mathcal{A}$ of projective objects.

Dually, we have

Lemma 2.4. Let $\mathcal{A}$ be an abelian category admitting enough injective objects. Let $X=\left(X^{i}, d^{i}\right)$ be a bounded complex in $\mathcal{A}$ such that the homology $H^{i}(X)$ has injective dimension at most $n$ for all $i$.Then $X \in\langle\mathcal{E}\rangle_{n+1} \subseteq D^{b}(\mathcal{A})$ for the subcategory $\mathcal{E} \subseteq \mathcal{A}$ of injective objects.

\subsection{Radical layer lengths and torsion pairs}

We recall some notions from [11. Let $\mathcal{C}$ be a length-category, that is, $\mathcal{C}$ is an abelian, skeletally small category and every object of $\mathcal{C}$ has a finite composition series. We use $\operatorname{End}_{\mathbb{Z}}(\mathcal{C})$ to denote the category of all additive functors from $\mathcal{C}$ to $\mathcal{C}$, and use rad to denote the Jacobson radical lying in $\operatorname{End}_{\mathbb{Z}}(\mathcal{C})$. For any $\alpha \in \operatorname{End}_{\mathbb{Z}}(\mathcal{C})$, set the $\alpha$-radical functor $F_{\alpha}:=\operatorname{rad} \circ \alpha$.

Definition 2.5. ([11, Definition 3.1]) For any $\alpha, \beta \in \operatorname{End}_{\mathbb{Z}}(\mathcal{C})$, we define the $(\alpha, \beta)$-layer length $\ell \ell_{\alpha}^{\beta}: \mathcal{C} \longrightarrow \mathbb{N} \cup\{\infty\}$ via $\ell \ell_{\alpha}^{\beta}(M)=\inf \left\{i \geqslant 0 \mid \alpha \circ \beta^{i}(M)=0\right\}$; and the $\alpha$-radical layer length $\ell \ell^{\alpha}:=\ell \ell_{\alpha}^{F_{\alpha}}$.

Lemma 2.6. Let $\alpha, \beta \in \operatorname{End}_{\mathbb{Z}}(\mathcal{C})$. For any $M \in \mathcal{C}$, if $\ell \ell_{\alpha}^{\beta}(M)=n$, then $\ell \ell_{\alpha}^{\beta}(M)=\ell \ell_{\alpha}^{\beta}\left(\beta^{j}(M)\right)+$ $j$ for any $0 \leqslant j \leqslant n$; in particular, if $\ell \ell^{\alpha}(M)=n$, then $\ell \ell^{\alpha}\left(F_{\alpha}^{n}(M)\right)=0$.

Proof. If $\ell \ell_{\alpha}^{\beta}(M)=n$, then $n=\inf \left\{i \geqslant 0 \mid \alpha \beta^{i}(M)=0\right\}$. By Definition 2.5, for any $0 \leqslant j \leqslant n$, we have

$$
\ell \ell_{\alpha}^{\beta}\left(\beta^{j}(M)\right)=\inf \left\{i \geqslant 0 \mid \alpha \beta^{i+j}(M)=0\right\}=n-j,
$$

that is, $\ell \ell_{\alpha}^{\beta}(M)=\ell \ell_{\alpha}^{\beta}\left(\beta^{j}(M)\right)+j$. In particular, if $\ell \ell^{\alpha}(M)=n$, then putting $\beta=F_{\alpha}$ we have $\ell \ell^{\alpha}\left(F_{\alpha}^{n}(M)\right)=\ell \ell^{\alpha}(M)-n=n-n=0$.

Recall that a torsion pair (or torsion theory) for $\mathcal{C}$ is a pair of classes $(\mathcal{T}, \mathcal{F})$ of objects in $\mathcal{C}$ satisfying the following conditions.

(1) $\operatorname{Hom}_{\mathcal{C}}(M, N)=0$ for any $M \in \mathcal{T}$ and $N \in \mathcal{F}$;

(2) an object $X \in \mathcal{C}$ is in $\mathcal{T}$ if $\left.\operatorname{Hom}_{\mathcal{C}}(X,-)\right|_{\mathcal{F}}=0$; 
(3) an object $Y \in \mathcal{C}$ is in $\mathcal{F}$ if $\left.\operatorname{Hom}_{\mathcal{C}}(-, Y)\right|_{\mathcal{T}}=0$.

For a subfunctor $\alpha$ of the identity functor $1_{\mathcal{C}}$, we write $q_{\alpha}:=1_{\mathcal{C}} / \alpha$. Let $(\mathcal{T}, \mathcal{F})$ be a torsion pair for $\mathcal{C}$. Recall that the torsion radical $t$ is a functor in $\operatorname{End}_{\mathbb{Z}}(\mathcal{C})$ such that

$$
0 \longrightarrow t(M) \longrightarrow M \longrightarrow q_{t}(M) \longrightarrow 0
$$

is a short exact sequence and $q_{t}(M)=M / t(M) \in \mathcal{F}$.

\section{Main results}

In this section, $\Lambda$ is an artin algebra. Then $\bmod \Lambda$ is a length-category. For a module $M$ in $\bmod \Lambda$, we use $\operatorname{rad} M, \operatorname{soc} M$ and top $M$ to denote the radical, socle and top of $M$ respectively. For a subclass $\mathcal{W}$ of $\bmod \Lambda$, we use add $\mathcal{W}$ to denote the subcategory of $\bmod \Lambda$ consisting of direct summands of finite direct sums of modules in $\mathcal{W}$, and if $\mathcal{W}=\{M\}$ for some $M \in \bmod \Lambda$, we write add $M:=\operatorname{add} \mathcal{W}$.

Let $\mathcal{S}$ be the set of all simple modules in $\bmod \Lambda$, and let $\mathcal{V}$ be a subset of $\mathcal{S}$ and $\mathcal{V}^{\prime}$ the set of all the others simple modules in $\bmod \Lambda$, that is, $\mathcal{V}^{\prime}=\mathcal{S} \backslash \mathcal{V}$. We write $\mathfrak{F}(\mathcal{V}):=\{M \in \bmod \Lambda \mid$ there exists a finite chain

$$
0=M_{0} \subseteq M_{1} \subseteq \cdots \subseteq M_{m}=M
$$

of submodules of $M$ such that each quotient $M_{i} / M_{i-1}$ is isomorphic to some module in $\mathcal{V}$ \}. By [11, Lemma 5.7 and Proposition 5.9], we have that $(\mathcal{T}, \mathfrak{F}(\mathcal{V}))$ is a torsion pair, where

$$
\mathcal{T}_{\mathcal{V}}=\left\{M \in \bmod \Lambda \mid \operatorname{top} M \in \operatorname{add} \mathcal{V}^{\prime}\right\}
$$

We use $t_{\mathcal{V}}$ to denote the torsion radical of the torsion pair $\left(\mathcal{T}_{\mathcal{V}}, \mathfrak{F}(\mathcal{V})\right)$. Then $t_{\mathcal{V}}(M) \in \mathcal{T}_{\mathcal{V}}$ and $q_{t_{\mathcal{V}}}(M) \in \mathfrak{F}(\mathcal{V})$ for any $M \in \bmod \Lambda$. By [11, Propositions 5.3 and $\left.5.9(\mathrm{a})\right]$, we have

\section{Proposition 3.1.}

(1) $\mathfrak{F}(\mathcal{V})=\left\{M \in \bmod \Lambda \mid t_{\mathcal{V}}(M)=0\right\}$;

(2) $\mathcal{T}_{\mathcal{V}}=\left\{M \in \bmod \Lambda \mid t_{\mathcal{V}}(M)=M\right\}$;

(3) top $M \in \operatorname{add} \mathcal{V}^{\prime}$ if and only if $t_{\mathcal{V}}(M)=M$.

As a consequence, we get the following

Proposition 3.2. If $\mathcal{V}=\varnothing$, then $\ell^{t \mathcal{V}}(M)=\operatorname{LL}(M)$ for any $M \in \bmod \Lambda$.

Proof. If $\mathcal{V}=\varnothing$, then the torsion pair $(\mathcal{T}, \mathfrak{F}(\mathcal{V}))=(\bmod \Lambda, 0)$. By Proposition $3.1(3)$, for any $M \in \bmod \Lambda$ we have $t_{\mathcal{V}}(M)=M$ and $\ell \ell^{t_{\mathcal{V}}}(M)=\mathrm{LL}(M)$.

\section{Lemma 3.3.}

(1) $\mathfrak{F}(\mathcal{V})$ is closed under extensions, submodules and quotient modules.

(2) The functor $t_{\mathcal{V}}$ preserves monomorphisms and epimorphisms. 
Proof. (1) It is [11, Lemma 5.7].

$(2)$ By [11, Lemma 3.6(a)], we have that $t_{\mathcal{V}}$ preserves monomorphisms. Since $\mathfrak{F}(\mathcal{V})$ is closed under quotient modules by (1), we have that $t_{\mathcal{V}}$ preserves epimorphisms by [4, Proposition $1.3]$.

We use $\mathbb{D}$ to denote the usual duality between $\bmod \Lambda$ and $\bmod \Lambda^{o p}$.

Proposition 3.4. Let $G$ be a generator and $E$ a cogenerator for $\bmod \Lambda$. Then $\ell \ell^{t} \mathcal{V}(G)=$ $\ell \ell^{t} \mathcal{V}(E)$. In particular, for any $M \in \bmod \Lambda$, we have

$$
\ell \ell^{t_{\mathcal{V}}}(M) \leqslant \ell \ell^{t_{\mathcal{V}}}(\Lambda)=\ell \ell^{t_{\mathcal{V}}}(\mathbb{D}(\Lambda))
$$

Proof. By Lemma 3.3(2) and [11, Lemma 3.4(b)(c)].

The following lemma is essentially contained in [14, Lemma 2.2.4]. A similar result also holds true for objects in the bounded derived category of a hereditary abelian category (see [12, 1.6] for details).

Lemma 3.5. Let

$$
X: \cdots \stackrel{d^{i-2}}{\longrightarrow} X^{i-1} \stackrel{d^{i-1}}{\longrightarrow} X^{i} \stackrel{d^{i}}{\longrightarrow} X^{i+1} \stackrel{d^{i+1}}{\longrightarrow} \cdots
$$

be a bounded complex in $\bmod \Lambda$ with all $X^{i}$ seimisimple. Then $X \cong \oplus_{i} H^{i}(X)[i]$ and $X \in$ $\langle\Lambda / \operatorname{rad} \Lambda\rangle$ in $D^{b}(\bmod \Lambda)$.

Proof. By assumption, there exist two integers $r$ and $t$ such that $X^{i} \in \operatorname{add}(\Lambda / \operatorname{rad} \Lambda)$, where $X^{i}=0$ for any $i \notin[r, t]$, where $[r, t]$ is the integer interval with endpoints $r$ and $t$. By [2, Theorem 9.6], the exact sequence

$$
0 \longrightarrow \operatorname{Ker} d^{t-1} \longrightarrow X^{t-1} \longrightarrow \operatorname{Im} d^{t-1} \longrightarrow 0
$$

splits. So the following complex

$$
0 \longrightarrow X^{r} \stackrel{d^{r}}{\longrightarrow} X^{r+1} \stackrel{d^{r+1}}{\longrightarrow} X^{r+2} \stackrel{d^{r+2}}{\longrightarrow} \cdots \stackrel{d^{t-2}}{\longrightarrow} X^{t-1} \stackrel{d^{t-1}}{\longrightarrow} X^{t} \longrightarrow 0
$$

is the direct sum of the following two complexes

$$
0 \longrightarrow X^{r} \stackrel{d^{r}}{\longrightarrow} X^{r+1} \stackrel{d^{r+1}}{\longrightarrow} X^{r+2} \stackrel{d^{r+2}}{\longrightarrow} \cdots \stackrel{d^{t-2}}{\longrightarrow} \text { Ker } d^{t-1} \longrightarrow 0 \longrightarrow 0
$$

and

$$
0 \longrightarrow 0 \longrightarrow 0 \longrightarrow 0 \longrightarrow \cdots \longrightarrow \operatorname{Im}^{t-1} \longrightarrow X^{t} \longrightarrow 0
$$

Note that the complex $(*)$ is isomorphic to the stalk complex $H^{t}(X)[t]$ in $D^{b}(\bmod \Lambda)$. By induction, we have $X \cong \oplus_{i=r}^{t} H^{i}(X)[i]$ in $D^{b}(\bmod \Lambda)$. 


\subsection{An upper bound for $\operatorname{dim} D^{b}(\bmod \Lambda)$}

We use $\mathcal{S}^{<\infty}$ to denote the set of the simple modules in $\bmod \Lambda$ with finite projective dimension, and use $\mathcal{S}^{\infty}$ to denote the set of the simple modules in $\bmod \Lambda$ with infinite projective dimension. Thus $\mathcal{S}^{<\infty} \cup \mathcal{S}^{\infty}=\mathcal{S}$. For a subset $\mathcal{V}$ of $\mathcal{S}$, it is easy to see that $\operatorname{pd} \mathfrak{F}(\mathcal{V}) \leqslant \operatorname{pd} \mathcal{V}$ and $\operatorname{id} \mathfrak{F}(\mathcal{V}) \leqslant$ id $\mathcal{V}$. We will use this observation in the sequel freely.

Lemma 3.6. Let $\mathcal{V}$ be a subset of $\mathcal{S}^{<\infty}$ and $\operatorname{pd} \mathcal{V}=a$. Then the following complex

$$
X: \cdots \stackrel{d^{i-2}}{\longrightarrow} X^{i-1} \stackrel{d^{i-1}}{\longrightarrow} X^{i} \stackrel{d^{i}}{\longrightarrow} X^{i+1} \stackrel{d^{i+1}}{\longrightarrow} \cdots
$$

with all $X^{i}$ in $\bmod \Lambda$ induces a complex

$$
q_{t_{\mathcal{V}}}(X): \cdots \stackrel{q_{t_{\mathcal{V}}}\left(d^{i-2}\right)}{\longrightarrow} q_{t_{\mathcal{V}}}\left(X^{i-1}\right) \stackrel{q_{t_{\mathcal{V}}}\left(d^{i-1}\right)}{\longrightarrow} q_{t_{\mathcal{V}}}\left(X^{i}\right) \stackrel{q_{t_{\mathcal{V}}}\left(d^{i}\right)}{\longrightarrow} q_{t_{\mathcal{V}}}\left(X^{i+1}\right) \stackrel{q_{t_{\mathcal{V}}}\left(d^{i+1}\right)}{\longrightarrow} \cdots
$$

such that $\operatorname{pd} H^{i}\left(q_{t_{\mathcal{V}}}(X)\right) \leqslant$ a for all $i$.

Proof. Since $q_{t_{\mathcal{V}}}$ is a covariant functor, we can obtain the complex $q_{t_{\mathcal{V}}}(X)$. For any $i$, since $q_{t_{\mathcal{V}}}\left(X^{i}\right) \in \mathfrak{F}(\mathcal{V})$, it follows from Lemma $3.3(1)$ that all $\operatorname{Ker} q_{t_{\mathcal{V}}}\left(d^{i}\right), \operatorname{Im} q_{t_{\mathcal{V}}}\left(d^{i-1}\right)$ and $H^{i}\left(q_{t_{\mathcal{V}}}(X)\right)$ are in $\mathfrak{F}(\mathcal{V})$. Thus we have $\operatorname{pd} H^{i}\left(q_{t_{\mathcal{V}}}(X)\right) \leqslant a$.

Lemma 3.7. Let $\mathcal{V}$ be a subset of $\mathcal{S}^{<\infty}$ and $\mathrm{pd} \mathcal{V}=a$. For a bounded complex $X=\left(X^{i}, d^{i}\right)$ in $\bmod \Lambda$, if $\ell \ell^{t_{\mathcal{V}}}(\Lambda)=n$, then $F_{t_{\mathcal{V}}}^{n}(X) \in\langle\Lambda\rangle_{a+1}$.

Proof. By Proposition 3.4, we have $\ell \ell^{t \mathcal{V}}\left(X^{i}\right) \leqslant \ell \ell^{t_{\mathcal{V}}}(\Lambda)=n$ for all $i$. Then by Lemma 2.6 and Proposition 3.1 (1), we have $\ell \ell^{t \mathcal{V}}\left(F_{t_{\mathcal{V}}}^{n}\left(X^{i}\right)\right)=0$ and $F_{t_{\mathcal{V}}}^{n}\left(X^{i}\right) \in \mathfrak{F}(\mathcal{V})$, which implies $H^{i}\left(F_{t_{\mathcal{V}}}^{n}(X)\right) \in$ $\mathfrak{F}(\mathcal{V})$ by Lemma $3.3(1)$, and hence $\operatorname{pd} H^{i}\left(F_{t_{\mathcal{V}}}^{n}(X)\right) \leqslant a$ for all $i$. It follows from Lemma 2.3 that $F_{t_{\mathcal{V}}}^{n}(X) \in\langle\Lambda\rangle_{a+1}$.

We now are in a position to prove the following

Theorem 3.8. Let $\mathcal{V}$ be a subset of $\mathcal{S}^{<\infty}$ and $\mathrm{pd} \mathcal{V}=a$. If $\ell^{t} \mathcal{V}(\Lambda)=n$, then

$$
\operatorname{dim} D^{b}(\bmod \Lambda) \leqslant(a+2)(n+1)-2
$$

Proof. If $\mathcal{V}=\varnothing$, then $\ell \ell^{t \mathcal{V}}(\Lambda)=\mathrm{LL}(\Lambda)$ by Proposition 3.2. Now the assertion follows from Theorem 1.1(1).

If $n=0$, that is, $t_{\mathcal{V}}(\Lambda)=0$, then $\Lambda \in \mathfrak{F}(\mathcal{V})$ by Proposition $3.1(1)$. Since $\mathcal{V}$ contains every simple module by the definition of $\mathfrak{F}(\mathcal{V})$ and since the composition series of $\Lambda$ does, we have $\mathcal{V}=\mathcal{S}$ and gl.dim $\Lambda=a$. It follows from Theorem 1.1 $(2)$ that $\operatorname{dim} D^{b}(\bmod \Lambda) \leqslant a$.

Let $X \in D^{b}(\bmod \Lambda)$ and $n \geqslant 1$. Since both $q_{t_{\mathcal{V}}}$ and $t_{\mathcal{V}}$ are covariant functors, we have that

$$
0 \longrightarrow t_{\mathcal{V}}(X) \longrightarrow X \longrightarrow q_{t_{\mathcal{V}}}(X) \longrightarrow 0
$$

is a short exact sequence of complexes. For any $Y \in D^{b}(\bmod \Lambda)$, we have the following short exact sequence of complexes

$$
0 \longrightarrow \operatorname{rad} Y \longrightarrow Y \longrightarrow \operatorname{top} Y \longrightarrow 0
$$


Now by letting $Y=t_{\mathcal{V}}(X)$, we have

$$
\begin{aligned}
\langle X\rangle & \subseteq\left\langle t_{\mathcal{V}}(X)\right\rangle \diamond\left\langle q_{t_{\mathcal{V}}}(X)\right\rangle \\
& \subseteq\left\langle t_{\mathcal{V}}(X)\right\rangle \diamond\langle\Lambda\rangle_{a+1} \quad(\text { by Lemmas [3.6 and 2.3) } \\
& \subseteq\left\langle\operatorname{rad} t_{\mathcal{V}}(X)\right\rangle \diamond\left\langle\operatorname{top} t_{\mathcal{V}}(X)\right\rangle \diamond\langle\Lambda\rangle_{a+1} \\
& =\left\langle F_{t_{\mathcal{V}}}(X)\right\rangle \diamond\left\langle\operatorname{top} t_{\mathcal{V}}(X)\right\rangle \diamond\langle\Lambda\rangle_{a+1} \\
& \subseteq\left\langle F_{t_{\mathcal{V}}}(X)\right\rangle \diamond\langle\Lambda / \operatorname{rad} \Lambda\rangle \diamond\langle\Lambda\rangle_{a+1} \quad \text { (by Lemma 3.5) } \\
& \subseteq\left\langle F_{t_{\mathcal{V}}}(X)\right\rangle \diamond\langle\Lambda \oplus(\Lambda / \operatorname{rad} \Lambda)\rangle_{a+2} . \quad(\text { by Lemma 2.2) }
\end{aligned}
$$

By replacing $X$ with $F_{t \mathcal{V}}^{i}(X)$ for any $1 \leqslant i \leqslant n-1$, we get

$$
\langle X\rangle \subseteq\left\langle F_{t_{\mathcal{V}}}^{n}(X)\right\rangle \diamond\langle\Lambda \oplus(\Lambda / \operatorname{rad} \Lambda)\rangle_{n(a+2)} .
$$

By Lemma 3.7, we have $F_{t_{\mathcal{V}}}^{n}(X) \in\langle\Lambda\rangle_{a+1}$. Thus

$$
\langle X\rangle \subseteq\langle\Lambda \oplus(\Lambda / \operatorname{rad} \Lambda)\rangle_{(n+1)(a+2)-1}
$$

It follows that $D^{b}(\bmod \Lambda)=\langle\Lambda \oplus(\Lambda / \operatorname{rad} \Lambda)\rangle_{(a+2)(n+1)-1}$ and

$$
\operatorname{dim} D^{b}(\bmod \Lambda) \leqslant(a+2)(n+1)-2 .
$$

We use $\mathcal{S}_{i n j}^{<\infty}$ to denote the set of the simple modules in $\bmod \Lambda$ with finite injective dimension. The following two lemmas are dual to Lemmas 3.6 and 3.7 respectively, we omit their proofs.

Lemma 3.9. Let $\mathcal{V}$ be a subset of $\mathcal{S}_{\text {inj }}^{<\infty}$ and id $\mathcal{V}=c$. Then the following complex

$$
X: \cdots \stackrel{d^{i-2}}{\longrightarrow} X^{i-1} \stackrel{d^{i-1}}{\longrightarrow} X^{i} \stackrel{d^{i}}{\longrightarrow} X^{i+1} \stackrel{d^{i+1}}{\longrightarrow} \cdots
$$

with all $X^{i}$ in $\bmod \Lambda$ induces a complex

$$
q_{t_{\mathcal{V}}}(X): \cdots \stackrel{q_{t_{\mathcal{V}}}\left(d^{i-2}\right)}{\longrightarrow} q_{t_{\mathcal{V}}}\left(X^{i-1}\right) \stackrel{q_{t_{\mathcal{V}}}\left(d^{i-1}\right)}{\longrightarrow} q_{t_{\mathcal{V}}}\left(X^{i}\right) \stackrel{q_{t_{\mathcal{V}}}\left(d^{i}\right)}{\longrightarrow} q_{t_{\mathcal{V}}}\left(X^{i+1}\right) \stackrel{q_{t_{\mathcal{V}}}\left(d^{i+1}\right)}{\longrightarrow} \cdots
$$

such that id $H^{i}\left(q_{t_{\mathcal{V}}}(X)\right) \leqslant c$ for all $i$.

Lemma 3.10. Let $\mathcal{V}$ be a subset of $\mathcal{S}_{i n j}^{<\infty}$ and $\mathrm{id} \mathcal{V}=c$. For a bounded complex $X=\left(X^{i}, d^{i}\right)$ in $\bmod \Lambda$, if $\ell \ell^{t_{\mathcal{V}}}(\mathbb{D}(\Lambda))=n$, then $F_{t_{\mathcal{V}}}^{n}(X) \in\langle\mathbb{D}(\Lambda)\rangle_{c+1}$.

The following result is dual to Theorem 3.8 .

Theorem 3.11. Let $\mathcal{V}$ be a subset of $\mathcal{S}_{\text {inj }}^{<\infty}$ and $\operatorname{id} \mathcal{V}=c$. If $\ell \ell^{t \mathcal{V}}(\mathbb{D}(\Lambda))=n$, then

$$
\operatorname{dim} D^{b}(\bmod \Lambda) \leqslant(c+2)(n+1)-2 .
$$

Proof. Though the proof is similar to that of Theorem 3.8, we still give it here for the readers' convenience.

If $\mathcal{V}=\varnothing$, then $\ell \ell^{t \mathcal{V}}(\mathbb{D}(\Lambda))=\operatorname{LL}(\mathbb{D}(\Lambda))=\operatorname{LL}(\Lambda)$ by Proposition 3.2. Now the assertion follows from Theorem 1.1(1). 
If $n=0$, that is, $t_{\mathcal{V}}(\mathbb{D}(\Lambda))=0$, then $\mathbb{D}(\Lambda) \in \mathfrak{F}(\mathcal{V})$ by Proposition $3.1(1)$. Since $\mathcal{V}$ contains every simple module by the definition of $\mathfrak{F}(\mathcal{V})$ and since the composition series of $\mathbb{D}(\Lambda)$ does, we have $\mathcal{V}=\mathcal{S}$ and gl.dim $\Lambda=c$. It follows from Theorem 1.1 (2) that $\operatorname{dim} D^{b}(\bmod \Lambda) \leqslant c$.

Let $X, Y \in D^{b}(\bmod \Lambda)$ and $n \geqslant 1$. Just like the argument in Theorem 3.8 , we have the following two short exact sequence of complexes

$$
\begin{gathered}
0 \longrightarrow t_{\mathcal{V}}(X) \longrightarrow X \longrightarrow q_{t_{\mathcal{V}}}(X) \longrightarrow 0 \\
0 \longrightarrow \operatorname{rad} Y \longrightarrow Y \longrightarrow \operatorname{top} Y \longrightarrow 0
\end{gathered}
$$

Now by letting $Y=t_{\mathcal{V}}(X)$, we have

$$
\begin{aligned}
\langle X\rangle & \subseteq\left\langle t_{\mathcal{V}}(X)\right\rangle \diamond\left\langle q_{t_{\mathcal{V}}}(X)\right\rangle \\
& \subseteq\left\langle t_{\mathcal{V}}(X)\right\rangle \diamond\langle\mathbb{D}(\Lambda)\rangle_{c+1} \quad(\text { by Lemmas }[3.9 \text { and 2.4) } \\
& \subseteq\left\langle\operatorname{rad} t_{\mathcal{V}}(X)\right\rangle \diamond\left\langle\operatorname{top} t_{\mathcal{V}}(X)\right\rangle \diamond\langle\mathbb{D}(\Lambda)\rangle_{c+1} \\
& =\left\langle F_{t_{\mathcal{V}}}(X)\right\rangle \diamond\left\langle\operatorname{top} t_{\mathcal{V}}(X)\right\rangle \diamond\langle\mathbb{D}(\Lambda)\rangle_{c+1} \\
& \subseteq\left\langle F_{t_{\mathcal{V}}}(X)\right\rangle \diamond\langle\Lambda / \operatorname{rad} \Lambda\rangle \diamond\langle\mathbb{D}(\Lambda)\rangle_{c+1} \quad(\text { by Lemma 3.5) } \\
& \subseteq\left\langle F_{t_{\mathcal{V}}}(X)\right\rangle \diamond\langle\mathbb{D}(\Lambda) \oplus(\Lambda / \operatorname{rad} \Lambda)\rangle_{c+2} . \quad \text { (by Lemma 2.2) }
\end{aligned}
$$

By replacing $X$ with $F_{t_{\mathcal{V}}}^{i}(X)$ for any $1 \leqslant i \leqslant n-1$, we get

$$
\langle X\rangle \subseteq\left\langle F_{t_{\mathcal{V}}}^{n}(X)\right\rangle \diamond\langle\mathbb{D}(\Lambda) \oplus(\Lambda / \operatorname{rad} \Lambda)\rangle_{n(c+2)} .
$$

By Lemma 3.10, we have $F_{t_{\mathcal{V}}}^{n}(X) \in\langle\mathbb{D}(\Lambda)\rangle_{c+1}$. Thus

$$
\langle X\rangle \subseteq\langle\mathbb{D}(\Lambda) \oplus(\Lambda / \operatorname{rad} \Lambda)\rangle_{(n+1)(c+2)-1} .
$$

It follows that $D^{b}(\bmod \Lambda)=\langle\mathbb{D}(\Lambda) \oplus(\Lambda / \operatorname{rad} \Lambda)\rangle_{(c+2)(n+1)-1}$ and

$$
\operatorname{dim} D^{b}(\bmod \Lambda) \leqslant(c+2)(n+1)-2 .
$$

Combining Theorems 3.8 and 3.11, we get the following

Theorem 3.12. Let $\mathcal{V}$ be a subset of $\mathcal{S}$ and $\min \{\operatorname{pd} \mathcal{V}, \operatorname{id} \mathcal{V}\}=d$. If $\ell \ell^{t} \mathcal{V}(\Lambda)=n$, then

$$
\operatorname{dim} D^{b}(\bmod \Lambda) \leqslant(d+2)(n+1)-2
$$

Proof. The case for $d=\infty$ is trivial. Since $\ell \ell^{t} \mathcal{V}(\Lambda)=\ell \ell^{t} \mathcal{V}(\mathbb{D}(\Lambda))$ by Proposition 3.4, the case for $d<\infty$ follows from Theorems 3.8 and 3.11 .

\subsection{An upper bound for $\operatorname{dim} D_{s g}^{b}(\bmod \Lambda)$}

Recall that the singularity category $D_{s g}^{b}(\bmod \Lambda)$ of $\bmod \Lambda$ is defined as $D^{b}(\bmod \Lambda) / K^{b}(\operatorname{proj} \Lambda)$, where $K^{b}(\operatorname{proj} \Lambda)$ is the bounded homotopy category of the subcategory $\operatorname{proj} \Lambda$ of $\bmod \Lambda$ consisting of projective modules. For any $M \in \bmod \Lambda$ and $m \geqslant 1$, we use $\Omega^{m}(M)$ to denote the $m$-th syzygy of $M$; in particular, $\Omega^{0}(M)=M$. 


\section{Lemma 3.13.}

(1) $\ell \ell^{t_{\mathcal{S}}<\infty}(\Lambda)=0$ if and only if $\operatorname{gl} \cdot \operatorname{dim} \Lambda<\infty$;

(2) $\ell \ell^{t_{\mathcal{S}}<\infty}(\Lambda) \neq 1$.

Proof. (1) If $\ell \ell^{t} \mathcal{S}^{<\infty}(\Lambda)=0$, then $t_{\mathcal{S}<\infty}(\Lambda)=0$. So $\Lambda \in \mathfrak{F}\left(\mathcal{S}^{<\infty}\right)$ by Proposition 3.1(1), which implies $\mathcal{S}^{<\infty}=\mathcal{S}$. Thus gl.dim $\Lambda=\operatorname{pd} \mathcal{S}=\operatorname{pd} \mathcal{S}^{<\infty}<\infty$. Conversely, if gl.dim $\Lambda<\infty$, then $\mathcal{S}^{<\infty}=\mathcal{S}$ and the torsion pair $\left(\mathcal{T}_{\mathcal{S}}<\infty, \mathfrak{F}\left(\mathcal{S}^{<\infty}\right)\right)=\left(\mathcal{T}_{\mathcal{S}}, \mathfrak{F}(\mathcal{S})\right)=(0, \bmod \Lambda)$. By Proposition 3.1(2), for any $M \in \bmod \Lambda$ we have $t_{\mathcal{S}<\infty}(M)=0$ and $\ell \ell^{t_{\mathcal{S}}<\infty}(\Lambda)=0$.

(2) Suppose $\ell \ell^{t}<\infty(\Lambda)=1$. Then by (1), we have gl.dim $\Lambda=\infty$ and there exists a simple module $S$ in $\bmod \Lambda$ such that $\operatorname{pd} S=\infty$. Consider the following exact sequence

$$
0 \longrightarrow \Omega^{1}(S) \longrightarrow P \longrightarrow S \longrightarrow 0
$$

in $\bmod \Lambda$ with $P$ the projective cover of $S$. Because top $S=S \in \operatorname{add} \mathcal{S}^{\infty}$, we have $t_{\mathcal{S}}<\infty(S)=S$ by Proposition 3.1(3). It follows from [11, Lemma 6.3] that

$$
\ell \ell^{t_{\mathcal{S}}<\infty}\left(\Omega^{1}(S)\right)=\ell \ell^{t_{\mathcal{S}}<\infty}\left(\Omega^{1}\left(t_{\mathcal{S}}<\infty(S)\right)\right) \leqslant \ell \ell^{t_{\mathcal{S}}<\infty}(\Lambda)-1=0,
$$

that is, $\ell \ell^{t} \mathcal{S}^{<\infty}\left(\Omega^{1}(S)\right)=0$, and $\Omega^{1}(S) \in \mathfrak{F}\left(\mathcal{S}^{<\infty}\right)$, which induces pd $\Omega^{1}(S)<\infty$, a contradiction.

In the following result, we give an upper bound for $\operatorname{dim} D_{s g}^{b}(\bmod \Lambda)$.

Theorem 3.14. Let $\mathcal{V}$ be a subset of $\mathcal{S}^{<\infty}$ with $\ell \ell^{t} \mathcal{V}(\Lambda)=n$. Then we have

$$
\operatorname{dim} D_{s g}^{b}(\bmod \Lambda) \leqslant \max \{0, n-2\}
$$

Proof. If $\mathcal{V}=\varnothing$, then $\ell \ell^{t \mathcal{V}}(\Lambda)=\operatorname{LL}(\Lambda)$ by Proposition 3.2. Now the assertion follows from Theorem 1.1(3).

Now suppose $\mathcal{V} \neq \varnothing$. If $n \leqslant 1$, then $\ell \ell^{t_{\mathcal{S}}<\infty}(\Lambda) \leqslant 1$ by [11, Proposition 5.10]. So $\ell \ell^{t_{\mathcal{S}}<\infty}(\Lambda)=$ 0 and gl.dim $\Lambda<\infty$ by Lemma 3.13, which implies $\operatorname{dim} D_{s g}^{b}(\bmod \Lambda)=0$.

Let $n \geqslant 2$ and set $a:=\operatorname{pd} \mathcal{V}$. From [8, Lemma 2.4(2)(a)], we know that every object in $D_{s g}^{b}(\bmod \Lambda)$ is isomorphic to a stalk complex for some module. Let $X \in \bmod \Lambda$. If $\ell \ell^{t \mathcal{V}}(X)=0$, then $\operatorname{pd} X<\infty$ and $X=0$ in $D_{s g}^{b}(\bmod \Lambda)$. If $\ell \ell^{t \mathcal{v}}(X)>0$, then by [11, Lemma 6.3], we have $\ell \ell^{t_{\mathcal{V}}}\left(\Omega^{1}\left(t_{\mathcal{V}}(X)\right)\right) \leqslant \ell \ell^{t_{\mathcal{V}}}(\Lambda)-1=n-1$. By Lemma 2.6. we have $\ell \ell^{t_{\mathcal{V}}}\left(F_{t_{\mathcal{V}}}^{n-1}\left(\Omega^{1}\left(t_{\mathcal{V}}(X)\right)\right)\right)=0$. By Proposition $3.1(1)$, we have $F_{t_{\mathcal{V}}}^{n-1}\left(\Omega^{1}\left(t_{\mathcal{V}}(X)\right)\right) \in \mathfrak{F}(\mathcal{V})$ and $\operatorname{pd} F_{t_{\mathcal{V}}}^{n-1}\left(\Omega^{1}\left(t_{\mathcal{V}}(X)\right)\right) \leqslant a$.

For any $Y \in \bmod \Lambda$, we have the following two exact sequences

$$
\begin{gathered}
0 \longrightarrow t_{\mathcal{V}}(Y) \longrightarrow Y \longrightarrow q_{t_{\mathcal{V}}}(Y) \longrightarrow 0, \\
0 \longrightarrow F_{t_{\mathcal{V}}}(Y) \longrightarrow t_{\mathcal{V}}(Y) \longrightarrow \operatorname{top} t_{\mathcal{V}}(Y) \longrightarrow 0 .
\end{gathered}
$$

Since $q_{t_{\mathcal{V}}}(Y) \in \mathfrak{F}(\mathcal{V})$, we have $\operatorname{pd} q_{t_{\mathcal{V}}}(Y) \leqslant a$. By the horseshoe lemma, we have

$$
\begin{gathered}
\Omega^{a+1}(Y) \cong \Omega^{a+1}\left(t_{\mathcal{V}}(Y)\right), \\
0 \rightarrow \Omega^{a+1}\left(F_{t_{\mathcal{V}}}(Y)\right) \rightarrow \Omega^{a+1}\left(t_{\mathcal{V}}(Y)\right) \oplus P_{1} \rightarrow \Omega^{a+1}\left(\operatorname{top} t_{\mathcal{V}}(Y)\right) \rightarrow 0,
\end{gathered}
$$


where $P_{1}$ is projective in $\bmod \Lambda$. Thus we have

$$
\begin{aligned}
\left\langle\Omega^{a+1}(Y)\right\rangle=\left\langle\Omega^{a+1}\left(t_{\mathcal{V}}(Y)\right)\right\rangle & \subseteq\left\langle\Omega^{a+1}\left(F_{t_{\mathcal{V}}}(Y)\right)\right\rangle \diamond\left\langle\Omega^{a+1}\left(\operatorname{top} t_{\mathcal{V}}(Y)\right)\right\rangle \\
& \subseteq\left\langle\Omega^{a+1}\left(F_{t_{\mathcal{V}}}(Y)\right)\right\rangle \diamond\left\langle\Omega^{a+1}(\Lambda / \operatorname{rad} \Lambda)\right\rangle .
\end{aligned}
$$

By replacing $Y$ with $F_{t_{\mathcal{V}}}^{i}(Y)$ for any $1 \leqslant i \leqslant n-2$, we get

$$
\left\langle\Omega^{a+1}(Y)\right\rangle \subseteq\left\langle\Omega^{a+1}\left(F_{t_{\mathcal{V}}}^{n-1}(Y)\right)\right\rangle \diamond\left\langle\Omega^{a+1}(\Lambda / \operatorname{rad} \Lambda)\right\rangle_{n-1} .
$$

Let $Y=\Omega^{1}\left(t_{\mathcal{V}}(X)\right)$. Since pd $F_{t_{\mathcal{V}}}^{n-1}\left(\Omega^{1}\left(t_{\mathcal{V}}(X)\right)\right) \leqslant a$, we have

$$
\Omega^{a+1}\left(F_{t_{\mathcal{V}}}^{n-1}\left(\Omega^{1}\left(t_{\mathcal{V}}(X)\right)\right)\right)=0,
$$

and so

$$
\left\langle\Omega^{a+2}\left(t_{\mathcal{V}}(X)\right)\right\rangle \subseteq\left\langle\Omega^{a+1}(\Lambda / \operatorname{rad} \Lambda)\right\rangle_{n-1} .
$$

By [8, Lemma $2.4(2)(\mathrm{b})]$, we have $X \cong \Omega^{a+2}(X)[a+2]$ in $D_{s g}^{b}(\bmod \Lambda)$. Thus

$$
X \cong \Omega^{a+2}(X)[a+2] \cong \Omega^{a+2}\left(t_{\mathcal{V}}(X)\right)[a+2] \in\left\langle\Omega^{a+1}(\Lambda / \operatorname{rad} \Lambda)\right\rangle_{n-1} .
$$

It follows that $D_{s g}^{b}(\bmod \Lambda)=\left\langle\Omega^{a+1}(\Lambda / \operatorname{rad} \Lambda)\right\rangle_{n-1}$ and $\operatorname{dim} D_{s g}^{b}(\bmod \Lambda) \leqslant n-2$.

The following corollary is an immediate consequence of Theorem 3.14. It is trivial that $\ell \ell^{t_{\mathcal{S}}<\infty}(\Lambda) \leqslant \operatorname{LL}(\Lambda)$, so this corollary improves Theorem 1.1(3).

Corollary 3.15. If $\ell^{t_{\mathcal{S}}<\infty}(\Lambda)=n$, then we have

$$
\operatorname{dim} D_{s g}^{b}(\bmod \Lambda) \leqslant \max \{0, n-2\}
$$

Now we explain why Theorem 1.1 is a special case of our results.

\section{Remark 3.16.}

(1) If $\mathcal{V}=\varnothing$, then $\ell \ell^{t} \mathcal{V}(\Lambda)=\operatorname{LL}(\Lambda)$ by Proposition 3.2. Since $c=\min \{\operatorname{pd} \mathcal{V}$, id $\mathcal{V}\}=-1$, by Theorem 3.12 we have

$$
\operatorname{dim} D^{b}(\bmod \Lambda) \leqslant(c+2)(n+1)-2=(-1+2)(\operatorname{LL}(\Lambda)+1)-2=\operatorname{LL}(\Lambda)-1 .
$$

This is Theorem 1.1(1).

By Theorem 3.14, we have

$$
\operatorname{dim} D_{s g}^{b}(\bmod \Lambda) \leqslant \max \{0, \operatorname{LL}(\Lambda)-2\}
$$

This is Theorem 1.1](3).

(2) If $\mathcal{V}=\mathcal{S}^{<\infty}=\mathcal{S}$, then the torsion pair $\left(\mathcal{T}_{\mathcal{V}}, \mathfrak{F}(\mathcal{V})\right)=(0, \bmod \Lambda)$. By Proposition $3.1(2)$, for any $M \in \bmod \Lambda$ we have $t_{\mathcal{V}}(M)=0$ and $\ell \ell^{t} \mathcal{V}(\Lambda)=0$. Because $c=\min \{\operatorname{pd} \mathcal{V}$, id $\mathcal{V}\}=$ gl.dim $\Lambda<\infty$, by Theorem 3.12 we have

$$
\operatorname{dim} D^{b}(\bmod \Lambda) \leqslant(c+2)\left(\ell \ell^{t \mathcal{V}}(\Lambda)+1\right)-2=(g \operatorname{ld} \operatorname{dim} \Lambda+2)(0+1)-2=\operatorname{gl} \cdot \operatorname{dim} \Lambda .
$$

This is Theorem 1.1(2). In addition, since gl.dim $\Lambda<\infty$, we have $\operatorname{dim} D_{s g}^{b}(\bmod \Lambda)=0$. 


\section{Examples}

By choosing some suitable $\mathcal{V}$ and applying Theorems 3.12 and 3.14, we may obtain more precise upper bounds for $\operatorname{dim} D^{b}(\bmod \Lambda)$ and $\operatorname{dim} D_{s g}^{b}(\bmod \Lambda)$ than that in Theorem 1.1. We give two examples to illustrate this. The global dimension of the algebra in the first example is infinite and that in the second one is finite.

Example 4.1. Consider the bound quiver algebra $\Lambda=k Q / I$, where $k$ is an algebraically closed field and $Q$ is given by

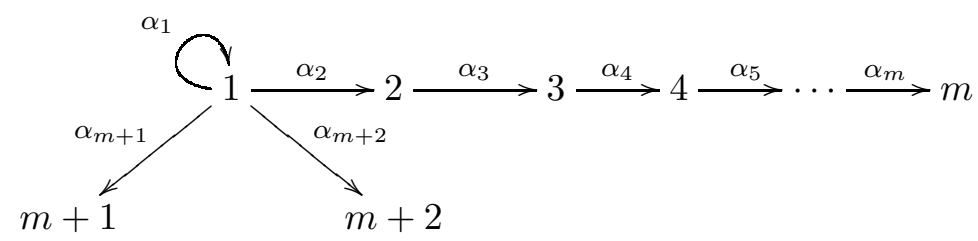

and $I$ is generated by $\left\{\alpha_{1}^{2}, \alpha_{1} \alpha_{m+1}, \alpha_{1} \alpha_{m+2}, \alpha_{1} \alpha_{2}, \alpha_{2} \alpha_{3} \cdots \alpha_{m}\right\}$ with $m \geq 10$. Then the indecomposable projective $\Lambda$-modules are

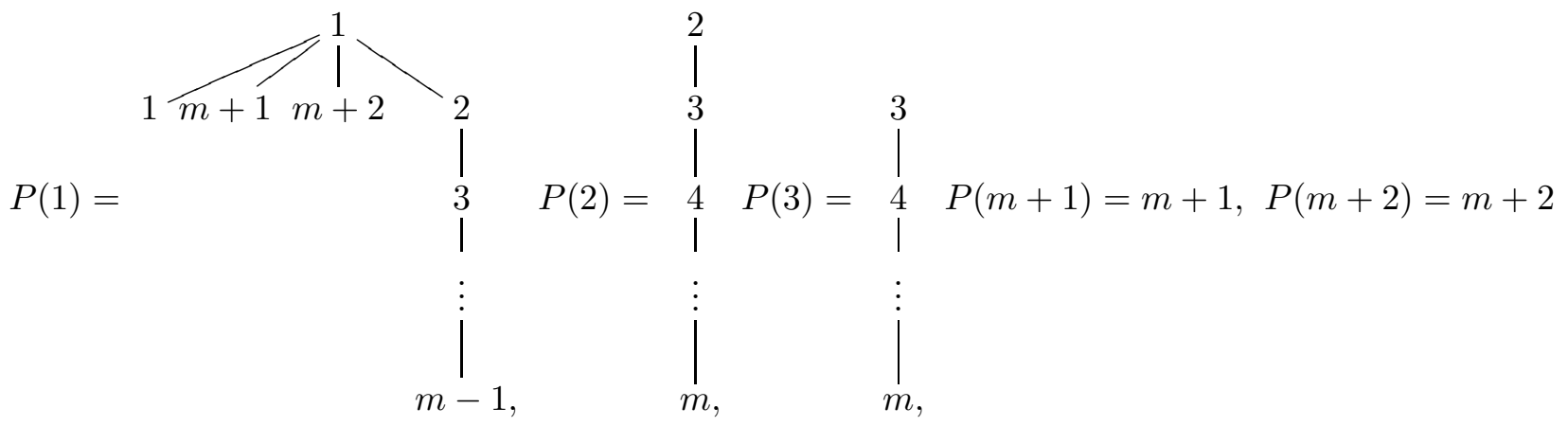

and $P(i+1)=\operatorname{rad} P(i)$ for any $2 \leqslant i \leqslant m-1$; and the indecomposable injective $\Lambda$-modules are

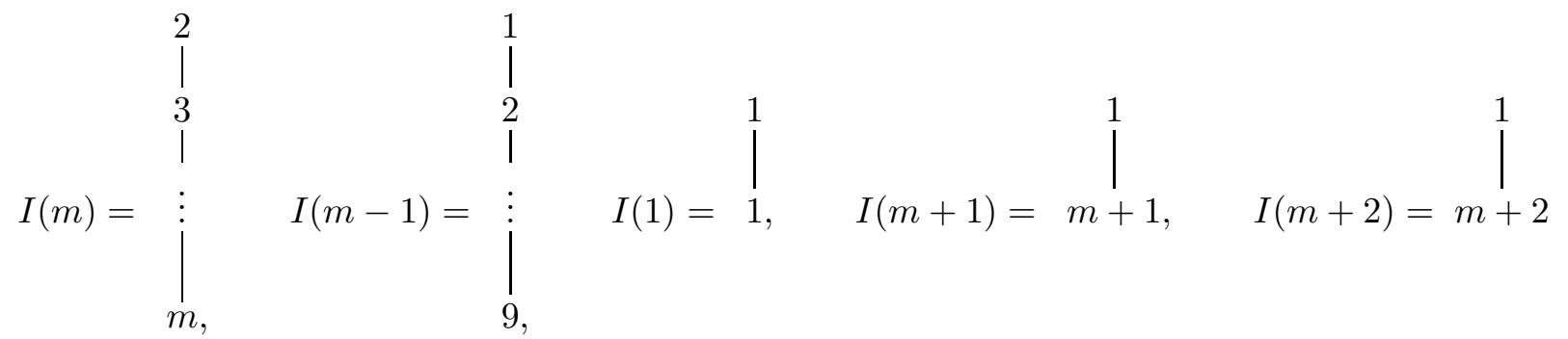

and $I(i)=I(i+1) / \operatorname{soc} I(i+1)$ for any $2 \leqslant i \leqslant m-2$.

We have

$$
\operatorname{pd} S(i)= \begin{cases}\infty, & \text { if } i=1 ; \\ 1, & \text { if } 2 \leqslant i \leqslant m-1 \\ 0, & \text { if } m \leqslant i \leqslant m+2 .\end{cases}
$$

So $\mathcal{S}^{\infty}=\{S(1)\}$ and $\mathcal{S}^{<\infty}=\{S(i) \mid 2 \leqslant i \leqslant m+2\}$. We also have

$$
\operatorname{id} S(i)= \begin{cases}\infty, & \text { if } i=1,2, m, m+1, m+2 \\ 1, & \text { if } 3 \leqslant i \leqslant m-1\end{cases}
$$


Let $\mathcal{V}:=\{S(i) \mid 3 \leqslant i \leqslant m-1\} \subseteq \mathcal{S}^{<\infty}$. Then

$$
a:=\operatorname{pd} \mathcal{S}=1, c:=\operatorname{id} \mathcal{S}=1 \text { and } d:=\min \{a, c\}=1 .
$$

Let $\mathcal{V}^{\prime}$ be all the others simple modules in $\bmod \Lambda$, that is, $\mathcal{V}^{\prime}=\{S(1), S(2), S(m), S(m+1), S(m+$ 2)\}. By [11, Lemma 3.4(a)] and $\Lambda=\oplus_{i=1}^{m+2} P(i)$, we have

$$
\ell \ell^{t \mathcal{V}}(\Lambda)=\max \left\{\ell \ell^{t \mathcal{V}}(P(i)) \mid 1 \leqslant i \leqslant m+2\right\} .
$$

In order to compute $\ell \ell^{t \mathcal{v}}(P(1))$, we need to find the least non-negative integer $i$ such that $t_{\mathcal{V}} F_{t_{\mathcal{V}}}^{i}(P(1))=0$. Since top $P(1)=S(1) \in \operatorname{add} \mathcal{V}^{\prime}$, we have $t_{\mathcal{V}}(P(1))=P(1)$ by Proposition 3.1(3). Thus

$$
F_{t_{\mathcal{V}}}(P(1))=\operatorname{rad} t_{\mathcal{V}}(P(1))=\operatorname{rad}(P(1))=S(1) \oplus S(m+1) \oplus S(m+2) \oplus T,
$$

where $T=\begin{gathered}2 \\ \mid \\ \vdots \\ \\ m-1 \\ m-1\end{gathered}$

Since top $S(1)=S(1) \in$ add $\mathcal{V}^{\prime}$, we have $t_{\mathcal{V}}(S(1))=S(1)$ by Proposition 3.1(3). Similarly, $t_{\mathcal{V}}(S(m+1))=S(m+1), t_{\mathcal{V}}(S(m+2))=S(m+2)$ and $t_{\mathcal{V}}(T)=T$. So

$$
t_{\mathcal{V}} F_{t_{\mathcal{V}}}(P(1))=t_{\mathcal{V}}(S(1) \oplus S(m+1) \oplus S(m+2) \oplus T)=S(1) \oplus S(m+1) \oplus S(m+2) \oplus T,
$$

and hence

$$
F_{t_{\mathcal{V}}}^{2}(P(1))=\operatorname{rad} t_{\mathcal{V}} F_{t_{\mathcal{V}}}(P(1))=\operatorname{rad}(S(1) \oplus S(m+1) \oplus S(m+2) \oplus T)=\operatorname{rad} T .
$$

It is easy to see that $\operatorname{rad} T \in \mathfrak{F}(\mathcal{V})$, so $t_{\mathcal{V}}(\operatorname{rad} T)=0$ by Proposition 3.1(1). Moreover, $t_{\mathcal{V}} F_{t_{\mathcal{V}}}^{2}(P(1))=0$. It follows that $\ell \ell^{t_{\mathcal{V}}}(P(1))=2$. Similarly, we have

$$
\ell \ell^{t \mathcal{V}}(P(i))= \begin{cases}2, & \text { if } i=2 \\ 1, & \text { if } 3 \leqslant i \leqslant m+2 .\end{cases}
$$

Thus $n:=\ell \ell^{t \mathcal{V}}(\Lambda)=\max \left\{\ell \ell^{t} \mathcal{V}(P(i)) \mid 1 \leqslant i \leqslant m+2\right\}=2$.

(1) Because $\operatorname{LL}(\Lambda)=m-1$, we have

$$
\operatorname{dim} D^{b}(\bmod \Lambda) \leqslant \operatorname{LL}(\Lambda)-1=m-2
$$

by Theorem 1.1(1). In particular, from Theorem 1.1(2), we can not get an upper bound for $\operatorname{dim} D^{b}(\bmod \Lambda)$. By Theorem 1.1)(3), we have

$$
\operatorname{dim} D_{s g}^{b}(\bmod \Lambda) \leqslant \operatorname{LL}(\Lambda)-2=m-3 .
$$

(2) By Theorem 3.12, we have

$$
\operatorname{dim} D^{b}(\bmod \Lambda) \leqslant(d+2)(n+1)-2=7 .
$$

By Theorem 3.14, we have

$$
\operatorname{dim} D_{s g}^{b}(\bmod \Lambda)=0 .
$$


Example 4.2. Consider the bound quiver algebra $\Lambda=k Q / I$, where $k$ is an algebraically closed field and $Q$ is given by

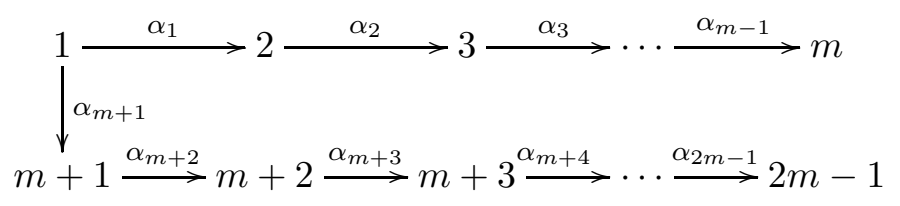

and $I$ is generated by $\left\{\alpha_{i} \alpha_{i+1} \mid m+1 \leqslant i \leqslant 2 m-2\right\}$ with $m \geqslant 9$. Then the indecomposable projective $\Lambda$-modules are

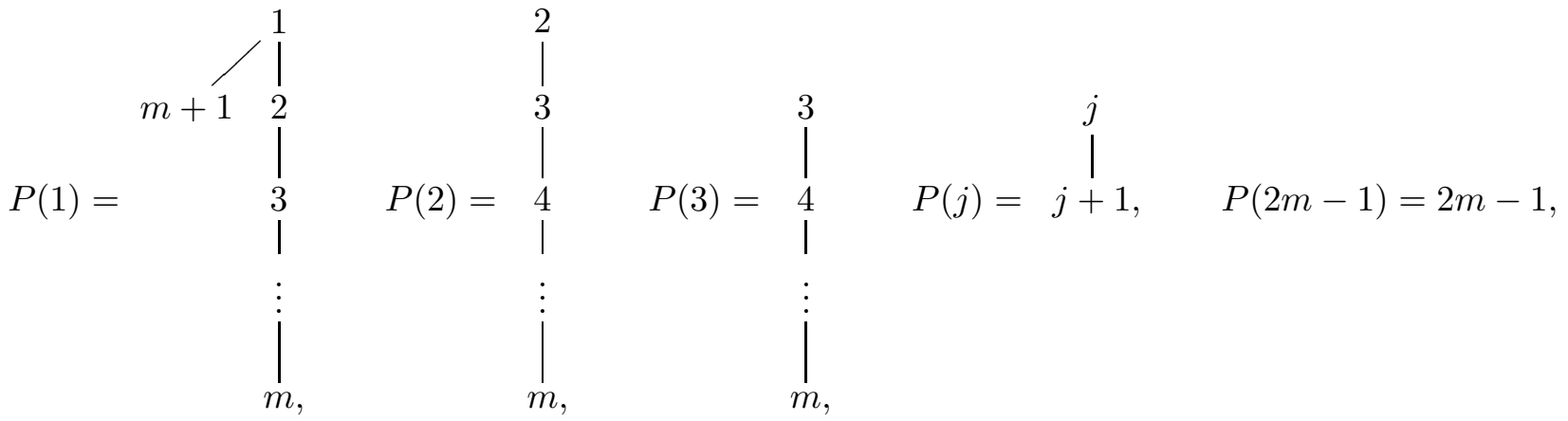

where $m+1 \leqslant j \leqslant 2 m-2$ and $P(i+1)=\operatorname{rad} P(i)$ for any $2 \leqslant i \leqslant m-1$; and the indecomposable injective $\Lambda$-modules are

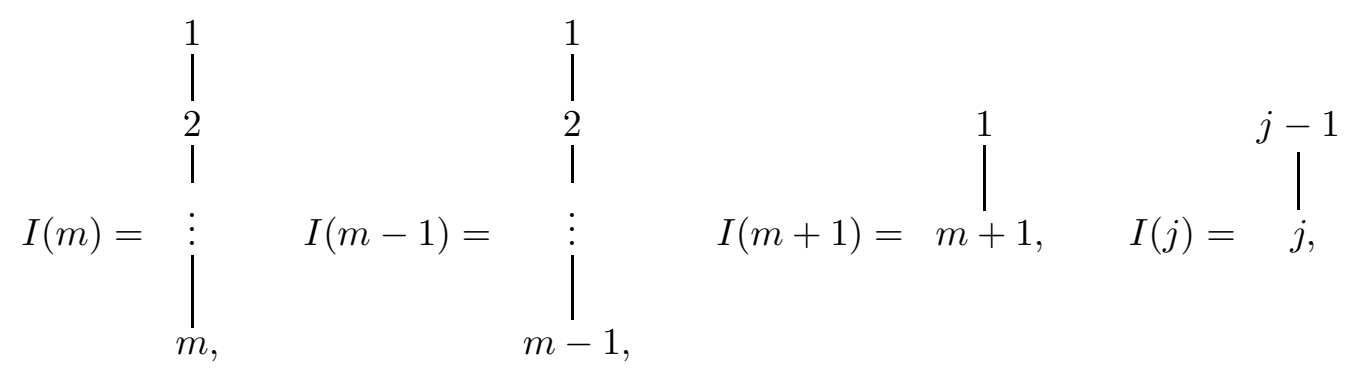

where $m+2 \leqslant j \leqslant 2 m-1$ and $I(i)=I(i+1) / \operatorname{soc} I(i+1)$ for any $1 \leqslant i \leqslant m-1$.

We have

$$
\operatorname{pd} S(i)= \begin{cases}m-1, & \text { if } i=1 \\ 1, & \text { if } 2 \leqslant i \leqslant m-1 \\ 0, & \text { if } i=m \\ 2 m-1-i, & \text { if } m+1 \leqslant i \leqslant 2 m-1\end{cases}
$$

and $\mathcal{S}^{<\infty}=\mathcal{S}$. We also have

$$
\operatorname{id} S(i)= \begin{cases}0, & \text { if } i=1 \\ 1, & \text { if } 2 \leqslant i \leqslant m ; \\ i-m, & \text { if } m+1 \leqslant i \leqslant 2 m-1 .\end{cases}
$$

Let $\mathcal{V}:=\{S(i) \mid 2 \leqslant i \leqslant m\} \subseteq \mathcal{S}^{<\infty}$. Then

$$
a:=\operatorname{pd} \mathcal{V}=1, c:=\operatorname{id} \mathcal{V}=1 \text { and } d:=\min \{a, c\}=1
$$


Let $\mathcal{V}^{\prime}$ be all the others simple modules in $\bmod \Lambda$, that is, $\mathcal{V}^{\prime}=\{S(i) \mid i=1$ or $m+1 \leqslant i \leqslant$ $2 m-1\}$. Similar to the computation in Example 4.1, we have $n:=\ell \ell^{t \mathcal{V}}(\Lambda)=2$.

(1) Because $\operatorname{LL}(\Lambda)=m$, we have

$$
\operatorname{dim} D^{b}(\bmod \Lambda) \leqslant \operatorname{LL}(\Lambda)-1=m-1
$$

by Theorem 1.1(1). Because gl.dim $\Lambda=m-1$, we also have

$$
\operatorname{dim} D^{b}(\bmod \Lambda) \leqslant \operatorname{gl} \cdot \operatorname{dim} \Lambda=m-1
$$

by Theorem 1.1(2). In addition, we have

$$
\operatorname{dim} D_{s g}^{b}(\bmod \Lambda) \leqslant \operatorname{LL}(\Lambda)-2=m-2
$$

by Theorem $1.1(3)$.

(2) By Theorem 3.12, we have

$$
\operatorname{dim} D^{b}(\bmod \Lambda) \leqslant(d+2)(n+1)-2=7
$$

By Theorem 3.14, we have

$$
\operatorname{dim} D_{s g}^{b}(\bmod \Lambda)=0
$$

In the above two examples, the upper bounds in (2) are smaller than that in (1) and the difference between them may be arbitrarily large.

Acknowledgements. This research was partially supported by National Natural Science Foundation of China (Grant Nos. 11971225, 11571164) and a Project Funded by the Priority Academic Program Development of Jiangsu Higher Education Institutions. The authors would like to thank Dong Yang for his helpful discussions, and thank the referees for very useful and detailed suggestions.

\section{References}

[1] T. Aihara and R. Takahashi, Generators and dimensions of derived categories of modules, Comm. Algebra 43 (2015), 5003-5029.

[2] F. W. Anderson and K. R. Fuller, Rings and Categories of Modules, Second edition, Grad. Texts in Math. 13, Springer-Verlag, New York, 1992.

[3] M. Ballard, D. Favero and L. Katzarkov, Orlov spectra: bounds and gaps, Invent. Math. 189 (2012), 359-430.

[4] J. A. Beachy, Cotorsion radicals and projective modules, Bull. Austral. Math. Soc. 5 (1971), $241-253$.

[5] P. A. Bergh, S. Oppermann, and D. A. Jorgensen, The Gorenstein defect category, Q. J. Math. 66 (2015), 459-471. 
[6] A. Bondal and M. van den Bergh, Generators and representability of functors in commutative and noncommutative geometry, Mosc. Math. J. 3 (2003), 1-36.

[7] X. W. Chen, Y. Ye and P. Zhang, Algebras of derived dimension zero, Comm. Algebra 36 (2008), 1-10.

[8] H. Dao and R. Takahashi, Upper bounds for dimensions of singularity categories, C. R. Math. Acad. Sci. Paris 353 (2015), 297-301.

[9] Y. Han, Derived dimensions of representation-finite algebras, Preprint is available at arXiv:0909.0330.

[10] F. Huard, M. Lanzilotta and O. Mendoza, Finitistic dimension through infinite projective dimension, Bull. Lond. Math. Soc. 41 (2009), 367-376.

[11] F. Huard, M. Lanzilotta and O. Mendoza Hernández, Layer lengths, torsion theories and the finitistic dimension, Appl. Categ. Structures 21 (2013), 379-392.

[12] H. Krause, Derived categories, resolutions, and Brown representability, Interactions between Homotopy Theory and Algebra, Contemp. Math. 436, Amer. Math. Soc., Providence, RI, 2007, pp.101-139.

[13] H. Krause and D. Kussin, Rouquier's theorem on representation dimension, Trends in Representation Theory of Algebras and Related Topics, Contemp. Math. 406, Amer. Math. Soc., Providence, RI, 2006, pp.95-103.

[14] S. Oppermann, Lower bounds for Auslander's representation dimension, Dissertation, Universität Köln, 2007.

[15] S. Oppermann and J. Št'ovíček, Generating the bounded derived category and perfect ghosts, Bull. Lond. Math. Soc. 44 (2012), 285-298.

[16] D. Orlov, Remarks on generators and dimensions of triangulated categories, Mosc. Math. J. 9 (2009), 153-159, back matter.

[17] C. Psaroudakis, Homological theory of recollements of abelian categories, J. Algebra 398 (2014), 63-110.

[18] R. Rouquier, Representation dimension of exterior algebras, Invent. Math. 165 (2006), $357-367$.

[19] R. Rouquier, Dimensions of triangulated categories, J. K-Theory 1 (2008), 193-256. 